\title{
Assessment of Post COVID-19 Rehabilitation Programme using Post-Acute Lung Injury Functional Scale
}

\author{
Nayana Gosavi ${ }^{1}$, Bhagyashri Karande ${ }^{2}$, Ajay Godse ${ }^{3}$, Sunil Jaisingh ${ }^{4}$ \\ ${ }^{1,2}$ Cardiopulmonary Physiotherapist, Working at Bhaktivedanta Hospital and Research Center \\ ${ }^{3,4}$ Chest Physician, Working at Bhaktivedanta Hospital and Research Center, Maharashtra.
}

Corresponding Author: Nayana Gosavi

\begin{abstract}
Background: Since its outbreak in China in January 2020, Coronavirus Disease 2019 (COVID-19) has spread worldwide and then, the World Health Organization declared that outbreak as a pandemic. The predominant pathological change is diffuse lung injury, most of the patients suffered from intraalveolar fibrinous exudates and pulmonary interstitial fibrosis, resulting in hypoxia, dyspnoea, and functional disabilities. Cardiopulmonary rehabilitation in COVID-19 patients is a nonpharmacological intervention which is still in evolving phase. Due to insufficient availability of data on functional assessment of any patient who has suffered a lung injury, this study has formulated a grading system for them. Also, we studied our patient data retrospectively to study the impact of cardiopulmonary rehabilitation in recovery of these patients.

Methods: This retrospective study was taken to do Functional assessment of patients with COVID-19 using PALIFS (Post-Acute Lung Injury Functional Scale). Along with formulating and executing individually tailored cardiopulmonary rehabilitation programme this study also focused on change in hemodynamic parameters, exercise tolerance and musculoskeletal strength before and after cardiopulmonary rehabilitation.

Results: A statistically significant difference ( $\mathrm{p}$ value $<0.0001$ ) was found in oxygen saturation level, heart rate at resting as well as post activity. Also, statistically significant difference in exercise tolerance and musculoskeletal strength post cardiopulmonary rehabilitation. Significantly slower recovery with advanced age but not with gender change and presence or absence of comorbidities.

Conclusion: Cardiopulmonary rehabilitation had a positive effect in recovery of COVID-19 patients. Also, the PALIFS was found to be an easy, effective, and appropriate tool for the functional assessment of COVID-19 patients.
\end{abstract}

Key words: COVID-19, Rehabilitation, Pulmonary, Functional scale, Lung injury

\section{INTRODUCTION}

Since its outbreak in China in January 2020, Coronavirus Disease 2019 (COVID-19) has spread worldwide and has become a global public health emergency. And then, the World Health Organization declared the outbreak a pandemic.

The primary clinical manifestations of COVID-19 are fever, cough, dyspnoea, and myalgia; ${ }^{1}$ however, severe cases can rapidly progress to acute respiratory distress syndrome (ARDS). In addition, some patients can develop acute myocardial and kidney injuries. ${ }^{2}$ The latest pathological reports indicate that the predominant pathological changes in early-3 and latestage patients are diffuse lung injuries, although some patients also suffered from intra-alveolar fibrinous exudates and pulmonary interstitial fibrosis. Moreover, the virus also affects other organs such as the heart, liver, and kidneys to various 
degrees. ${ }^{4}$ These changes contribute to hypoxemia and impaired cardiopulmonary and organ functions throughout the body. Currently, evidence on the prognosis of patients with COVID-19 is insufficient, especially for elderly patients in whom the disease is complicated by other basic diseases. It remains unclear whether the impairment of multiple systemic functions is reversible or if the long-term existence of the virus can cause physical dysfunction in these patients. In addition, because COVID19 has caused a public emergency, patients with COVID-19 may demonstrate different degrees of psychological disorders, such as anger, fear, anxiety, depression, insomnia, and loneliness, as well as a lack of cooperation and abandonment of treatment due to fear of the disease. ${ }^{5}$ Even when discharged, the patients may experience post-traumatic stress syndrome. Therefore, prompt introduction and continuous availability of pulmonary rehabilitation services is critical for patients with COVID19.

\section{Pulmonary Rehabilitation}

Pulmonary rehabilitation refers to the individualized rehabilitation treatment of patients with chronic pulmonary diseases after a detailed assessment. With exercise training as its core, pulmonary rehabilitation comprises comprehensive interventions, including but not limited to psychological and nutritional support, as well as education and behavioural changes. ${ }^{6}$ The goal of pulmonary rehabilitation is to not only improve the patient's physical and mental conditions but also help the patient return to family and society more promptly.

The benefits include improved exercise tolerance in patients with chronic pulmonary diseases, reduced number of hospital admissions and length of hospital stays, enhanced health-related quality of life,5 improved respiratory muscle function and relieved dyspnea, ${ }^{7}$ alleviated diseaserelated anxiety and depression, ${ }^{8}$ and enhanced skeletal muscle function of upper and lower limbs. ${ }^{9,10}$

\section{Evidence for Pulmonary Rehabilitation of Patients with SARS, COVID 19}

Follow-up studies have shown that after discharge, patients with severe acute respiratory syndrome (SARS) can still suffer from symptoms, such as restrictive pulmonary dysfunction, palpitations, hand tremors, and exertional dyspnoea, all of which affect their daily activities and impair their quality of life. ${ }^{11,12}$ It has been suggested that these symptoms are associated with prolonged bed rest, adverse effects of steroid medications, and residual pathological changes, such as atelectasis, persistent alveolitis, pulmonary fibrosis, and varying degrees of muscle weakness or dysfunction.13 In addition, a 1-year followup of patients with ARDS showed that, survivors of ARDS exhibit persistent functional disability one year after discharge from the intensive care unit. Most patients have extrapulmonary conditions, with muscle wasting and weakness being most prominent. ${ }^{14}$ Also, pathological changes such as pulmonary fibrosis, have been dominant in patients with COVID-19, we speculate that damage to the lung and other organ systems caused by SARSCoV-2, especially in severe patients with ARDS, may lead to residual physical dysfunction of varying degrees. Thus, the evidence for pulmonary rehabilitation of patients with SARS provides strong support and reference for the development of pulmonary rehabilitation programs for patients with COVID-19.

Need for the study: Cardiopulmonary rehabilitation in COVID-19 patients is a nonpharmacological intervention which is still in evolving phase. We got study material on the same, but it is insufficient on functional assessment of any patient who has suffered a lung injury due to any cause. Hence, we formulated a grading system for them which will include patient's mobility, oxygen dependency and ability to perform activities of daily living. Also, we studied our patient data retrospectively to study the 
effects of cardiopulmonary rehabilitation in these patients.

\section{MATERIALS}

- Pulse oximeter

- Digital Blood Pressure Equipment

- Stationary cycle/ Pedal Bedside cycle

- Oxygen supply

- Silicon tubing extension for oxygen supplementation
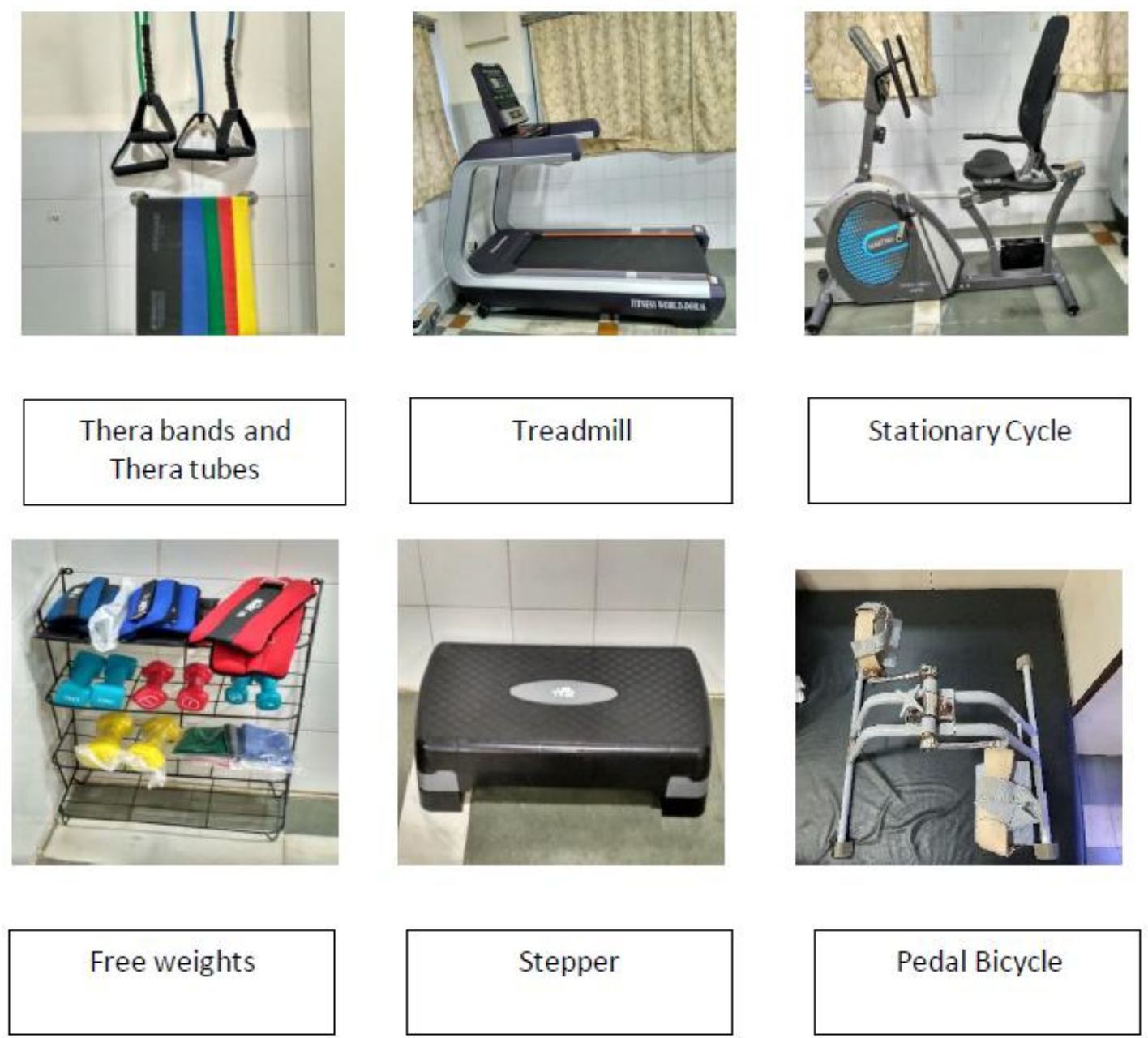

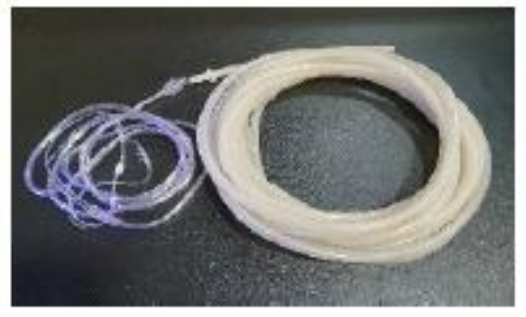

- Post-Acute lung injury Functional Scale:

The following grading system is made to classify patients as per their impairments and need for cardiopulmonary rehabilitation.

\section{Silicon tubes for} extension of oxygen 


\section{Post-Acute lung Injury Functional Scale}

\begin{tabular}{|l|l|}
\hline Grade 0 & No residual symptoms, $\mathrm{SpO}_{2}>95 \%$ on room air, Asymptomatic Symptom limited walk test \\
\hline Grade 1 & $\begin{array}{l}\text { Breathlessness/Fatigue during BADL but no need of Oxygen supplementation as } \mathrm{SpO}_{2}>95 \% \text {, can perform Symptom limited } \\
\text { walk test. }\end{array}$ \\
\hline Grade 2 & $\begin{array}{l}\text { Breathlessness/Fatigue during BADL, needs low flow oxygen supplementation to maintain } \mathrm{SpO}_{2}>95 \%, \text { can still perform } \\
\text { Symptom limited walk test. }\end{array}$ \\
\hline Grade 3 & $\begin{array}{l}\text { Comfortable with Low flow oxygen supplementation at rest but needs High flow oxygen supplementation during activities, } \\
\text { able to do bedside activities independently or with minimal support. }\end{array}$ \\
\hline Grade 4 & Needs Continuous High flow oxygen supplementation but able to do bedside activities with support. \\
\hline Grade 5 & $\begin{array}{l}\text { Needs intermittent/ continuous pressure support ventilation along with high flow oxygen supplementation, able to do in bed } \\
\text { activities. }\end{array}$ \\
\hline
\end{tabular}

This functional grading was assessed and scrutinized by the group of physicians in our institute and after the approval from our institutional ethics committee, we started using for our patient population which then got considered for this study protocol.

\section{METHODOLOGY}

- COVID-19 patients included from Last week Of May 2020 to second week of January-2021.

- As per the Study protocol Chest Physiotherapy sessions started after hemodynamic stability, an individually tailored graded exercise programme was formulated.

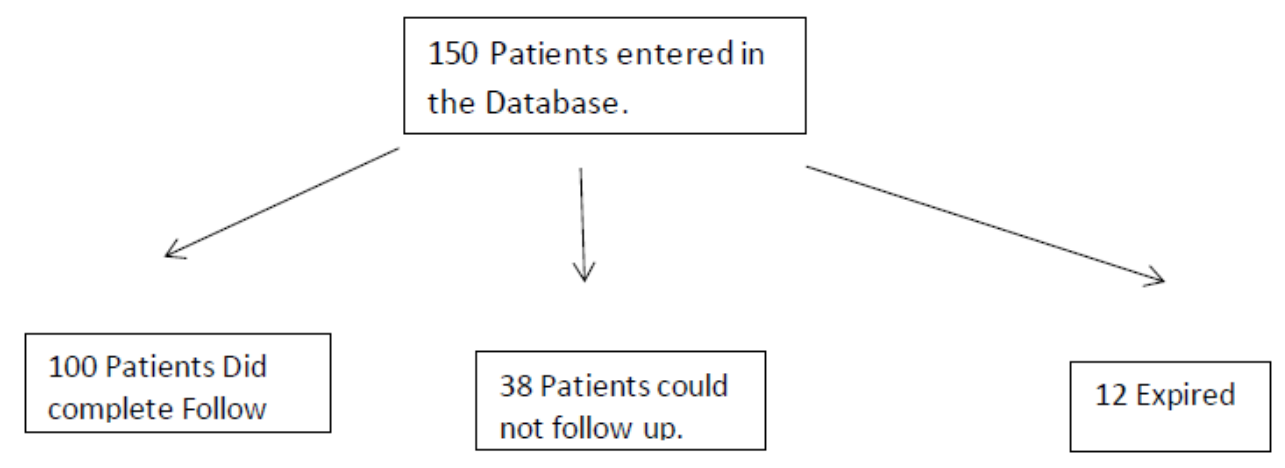

Hence, for this study Sample size we got it as 100 .

\section{Aim of the study:}

To study the impact of cardiopulmonary rehabilitation in recovery of COVID-19 patients.

\section{Type of Study: Retrospective}

\section{Objectives:}

1. To do Functional assessment of patients with COVID-19 using PALIFS on weekly basis.

2. Formulate and execute individually tailored cardiopulmonary rehabilitation program.

3. To study $\mathrm{SpO}_{2}$ and HR before and after cardiopulmonary Rehabilitation.
4. To study the change in exercise tolerance (duration) before and after cardiopulmonary rehabilitation.

5. To study the change in musculoskeletal strength before and after cardiopulmonary rehabilitation.

6. To study length of the hospital, stay with respect to age, gender, comorbidities, and early and late cardiopulmonary rehabilitation.

\section{Inclusion criteria:}

1. Age group: $18-90 \mathrm{yrs}$

2. All genders included

3. Cardiac stability- absence of arrythmias, no inotropic drugs required.

4. In case of NIV or Invasive ventilation, when $\mathrm{FiO}_{2}<50 \%$, PEEP/CPAP $<10 \mathrm{~cm}$ of $\mathrm{H}_{2} \mathrm{O}$. 
5. If patient had Pneumothorax, then after resolution of it with ICD or High Flow $\mathrm{O}_{2}$ therapy.

\section{Exclusion criteria:}

1. Unstable vitals.

2. Increase in breathlessness or signs of ARDS.

3. Untreated Pneumothorax or Pneumomediastinum.

4. Sudden chest pain.

5. Sudden increase in requirement of oxygen.

\section{Assessment:}

1. Hemodynamic markers: $\mathrm{O}_{2}$ saturation, Blood pressure and Heart rate

2. Post-Acute Lung Injury Functional Scale

3. Symptom limited walk test.

4. MMT for strength assessment (both Upper limb and Lower limb)

5. 1 Minute Sit to stand (Optional)

6. CT Scan/ X-ray-Chest for reference

\section{Physiotherapy intervention/}

Cardiopulmonary Rehabilitation:

To improve ventilation/ reduce anxiety/ improve oxygen saturation:

- Breathing control exercises.

- Breathing retraining.

- Dyspnea relieving positions.

- Positional rotation (supine but propped up/prone/side lying/sitting supported)

\section{Functional training:}

- Sitting unsupported in the bed

- Sitting at the edge of the bed with legs dangling
- Sit to stand from the bed.

- Standing unsupported

- Sit to stand in chair.

\section{Progressing it with Aerobic training:}

- Bedside walking with or without oxygen support

- Gradually increase the duration of walk

- Gradually reduce the oxygen requirement

- Cycling:

1. Bedside Pedal cycle

2. Progression with stationary cycle

3. Increase the duration then the Speed/RPM.

- Stepping with stepper: start with 5 then progression up to 30 steps.

To improve peripheral oxygen uptake/ strength training:

- General conditioning exercises with active range of motion exercises from distal to proximal joints.

- Progress from in bed to bedside or from supported to unsupported position.

- Use of free weights for upper limb from $0.5 \mathrm{~kg}$ to $1 \mathrm{~kg}$, progress from 5 reps to 20 reps.

- Use of Thera band and Thera tube exercises for upper back and shoulder muscles

- For lower limb strengthening, from AROM exercises to weight bearing exercises like heel raises, squatting, lunges and sit to stand from chair.

- Progression of weight cuffs for lower limb from $0.5 \mathrm{~kg}$ to $1 \mathrm{~kg}$ and then from 5reps to 20 .

\begin{tabular}{|l|l|l|}
\hline & IPD & OPD \\
\hline Intensity & Borg Scale3-4/10, $\mathrm{SpO}_{2}<3 \%$ & THR=40-60\%(HRR), $\mathrm{SpO}_{2}<4 \%$ \\
\hline Frequency & $1-2$ times/day & $2-3$ times/ week \\
\hline Duration & $10-45$ mins including rest period & $30-60 \mathrm{mins}$ \\
\hline Strength training & Low intensity (Conditioning exercises), Daily & low to moderate intensity, 2-3 times/Week \\
\hline
\end{tabular}

\section{Reassessment:}

- Hemodynamic measures $\left(\mathrm{SpO}_{2}, \mathrm{HR}\right.$, $\mathrm{RR}, \mathrm{BP}, \mathrm{O}_{2}$ requirement) taken on every session.

- PALIFS will be done every week.

\section{RESULT AND STATISTICAL}

ANALYSIS

Data analysis was done using the SPSS (Statistical Package for the Social Science) Version 23 for window. The 
demographic variables, PALIFS score, musculoskeletal strength, duration needed for recovery were calculated with number and percentage. The paired $t$ test was used to find significant differences of $\mathrm{SpO}_{2}$ minimum and maximum, HR minimum and maximum, exercise tolerance before and after cardiopulmonary rehabilitation and unpaired $t$ test was used to find the significant difference of length of stay between OPD and IPD, duration needed for recovery between male and females, duration needed for recovery between comorbidity present and absent. Paired t test was used to see significant difference of recovery time with respect to age. The chi square test was used to find the significant association of PALIFS score in OPD and IPD. A probability value of 0.05 was accepted as the level of statistical significance.

From our study population demographic details can be summarised as follows,

\begin{tabular}{|l|l|l|}
\hline Unit & OPD & $52 \%$ \\
\hline & IPD & $48 \%$ \\
\hline Gender & MALES & $72 \%$ \\
\hline & FEMALES & $28 \%$ \\
\hline Age groups & $30-40$ & $8 \%$ \\
\hline & $41-50$ & $10 \%$ \\
\hline & $51-60$ & $34 \%$ \\
\hline & $61-70$ & $32 \%$ \\
\hline & 71 and above & $16 \%$ \\
\hline
\end{tabular}

Also, the Post-Acute Lung Injury Functional Score,

\begin{tabular}{|l|l|l|l|}
\hline PALIFS score & IPD & OPD & $(\boldsymbol{\%})$ \\
\hline Grade 1 & 6 & 34 & 40 \\
\hline Grade 2 & 17 & 16 & 33 \\
\hline Grade 3 & 15 & 2 & 17 \\
\hline Grade 4 & 5 & 0 & 5 \\
\hline Grade 5 & 5 & 0 & 5 \\
\hline Total & 48 & 52 & 100 \\
\hline
\end{tabular}

Among the study population $48 \%$ of patients started their Rehab from ICU and ward (IPD) whereas 52\% of patient started their rehab on OPD basis. In IPD patients, Grade 1 patients were $6 \%$, Grade2 were $17 \%$, Grade 3 were $15 \%$, Grade 4 were $5 \%$ and Grade 5 were $5 \%$

Whereas out of $52 \%$ of OPD patients, Grade 1 were $34 \%$, Grade 2 were $16 \%$, Grade 3 were $2 \%$ and no patient of Grade 4 or 5 . And there was no statistically significant difference was found in length of stay of IPD and OPD patients.

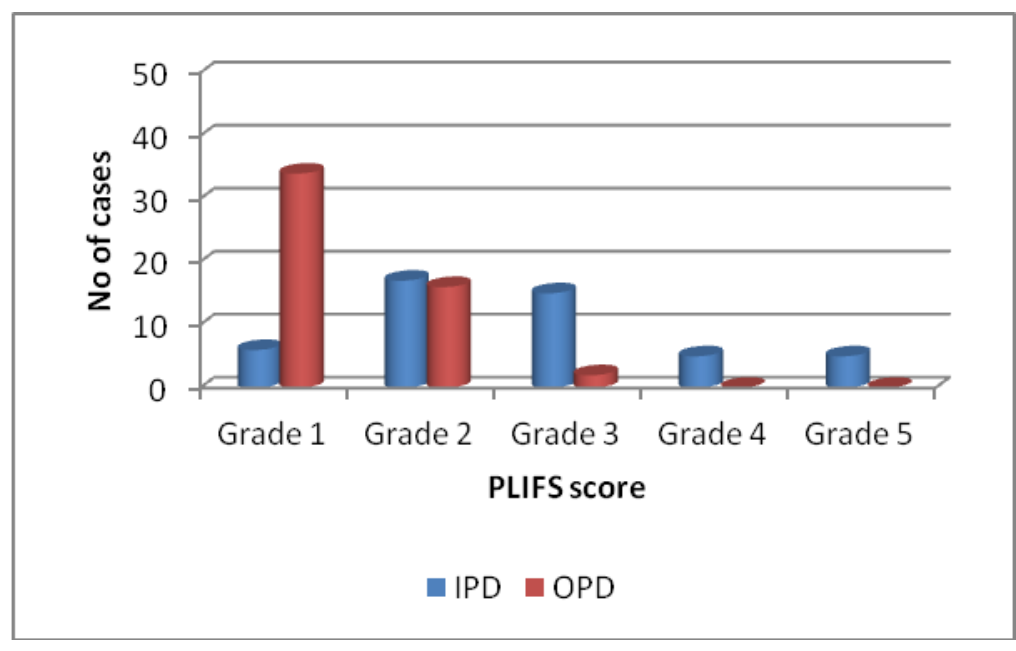

A statistically significant difference ( $p$ value $<0.0001$ ) was found in oxygen saturation level at resting as well as post activity. From minimum oxygen saturation level of $87.86 \%$ (mean value) it increased up to $94.20 \%$ (mean value) and maximum oxygen saturation level of $95.37 \%$ (mean value) increased up to $97.82 \%$ (mean value) 
A statistically significant difference ( $\mathrm{p}$ value $<0.0001$ ) was found in Heart Rate at resting as well as post activity. Resting as well as exertional tachycardia was seen less after cardiopulmonary rehabilitation. The resting heart rate reduced from $98.89 \mathrm{bpm}$ (mean value) to $84.03 \mathrm{bpm}$ (mean value) and post activity the heart rate changed from $127.76 \mathrm{bpm}$ (mean value) to $112.75 \mathrm{bpm}$ (mean value).

A statistically significant difference ( $p$ value $<0.0001$ ) was found in exercise tolerance, they could do basic activities of daily living with minimal or no exertion.
Their exercise duration increased from $11.08 \mathrm{mins}$ (mean value) to $66.06 \mathrm{mins}$ (mean value) including rest periods.

There was statistically significant difference ( $\mathrm{p}$ value <0.0001) found the strength of both upper limb (From 3-/5 to $5 / 5$ and lower limb muscles (from $2+/ 5$ to $5 / 5)$.

There was a statistically significant difference found in recovery time when considered age groups. ( $\mathrm{p}$ value $=0.01$ ) with advanced age the recovery time needed was more

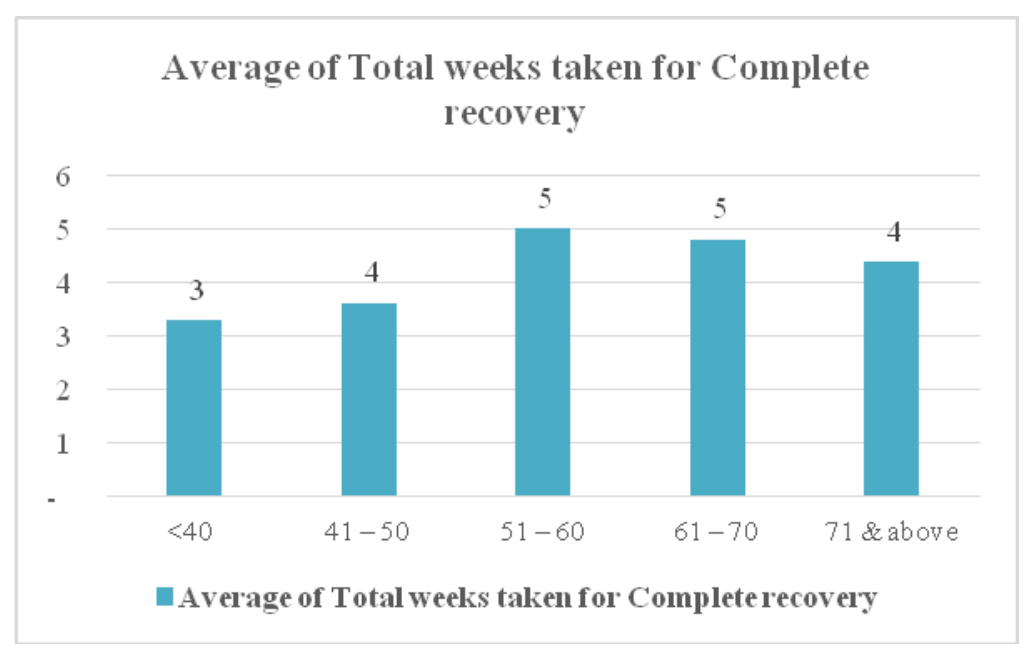

There was no statistically significant difference found in recovery time when considered gender ( $p$ value $=0.48)$ and presence or absence of comorbidities ( $p$ value $=0.50$ ).

There was no statistically significant difference found in length of hospital stay in OPD group and IPD group, $p$ value $=0.88$.

\section{DISCUSSION}

In ICU, after they attained hemodynamic stability chest physiotherapy interventions were started and progressed as per their activity tolerance whereas in OPD as per their functional assessment and need for oxygen, exercise programme is formulated and progressed.

Pulse Oximetry played a crucial role in deciding the safety of this intervention. There was no rule of thumb to determine target intensity. It was based on real time response of change in saturation with exercise or activity at least in the early phases of rehabilitation before the patient is eligible for a target heart rate exercise. Continuous monitoring of heart rate and oxygen saturation with pulse oximeter during exercise and in recovery phase was important. The mobilization was discontinued if saturation dropped more than $4 \%$ from resting or maximum of $87 \%$ and or if heart rate $>30$ beats from resting or maximum of 140 beats per minute. Low intensity graded functional and strengthening exercises with slow and sustained progression based on subjective measures like fatigue and breathlessness along with objective measures like HR, $\mathrm{SpO}_{2}$ and need of oxygen.

Aerobic training has the capacity to improve oxygen consumption by conditioning the skeletal and respiratory 
muscles for better oxygen uptake due to the physiologic changes in the structure and function of muscles at the cellular level 16 . Once the patient does not de-saturate with exercise and is hemodynamically stable, a target heart rate can be decided using the Karvonen's formula starting at 40 to $50 \%$ of heart rate reserve and then progress to higher intensity based on the response.

The pro-inflammatory markers like IL6, IL1, TNF alpha etc, representing the presence of multisystem inflammation, show a decrease with regular aerobic exercise and there is a rise in IL-10 which is an anti-inflammatory marker. Also, exercise induced regeneration and reendothelialisation of injured endothelium by increasing the number of circulating endothelial progenitor cell which would be beneficial in COVID-19 mediated endothelial apoptosis and endothelial cell membrane disruption. Antipathogenic activity of macrophages along with elevations in the circulation of immune cells, immunoglobulins and antiinflammatory cytokines which modulates and improves the immune response. This reduces the influx of inflammatory cells which reduces the risk of lung damage and the hypercoagulability of blood due to COVID-19.

Graded mobility exercises and functional training along with low to moderate intensity aerobic training improved oxygen transport at all levels. With improved peripheral oxygen uptake, it causes improvement in tissue perfusion of all organs ${ }_{16}$. This results in better oxygen saturation both at resting and post activity. As the cellular hypoxia recovers slowly, autonomic nervous system responds in a better way causing reduction in resting tachycardia 16 . With better saturation, reduced tachycardia, better functional mobility, the PALIFS Score also changed from Grade 5 to Grade 0.

Critical illness myopathies and polyneuropathies as well as steroid induced myopathies made patients more dependent on oxygen, loss of strength in proximal and postural or antigravity muscles, along with sarcopenia, osteopenia/ osteoporosis was also seen $_{17,18}$. Low to moderate intensity strengthening exercises helped to maintain and combat the muscle loss by improving its fitness and ultimately improving peripheral oxygen uptake and muscle physiology, preventing muscle atrophy ${ }_{18}$.

A significant change was seen in recovery time when considered age. As with advanced age the recovery took a longer time, irrespective of the functional grade of the person and rehab group (IPD/OPD). From a large study including 1,068 healthy adults, it was shown that the circulating naïve CD4+ and CD8+ $\mathrm{T}$ cells would decrease with aging, which is a sign of immunosenescence of the immune system 19 . There is age related decline in chest wall compliance along with senile emphysema which reduces oxygenation. Loss of fast twitch fibres of diaphragm causes early fatigue and ventilatory failure during increased ventilatory load on pulmonary system. Additional age-related changes including reduced heart rate response, cardiac output and peripheral muscle mass may account for the decline in $\mathrm{VO}_{2} \max$ with age rather than reduced lung function alone $_{20}$.

No significant change in recovery with respect to gender and comorbidities was observed.

Out of 100 patients 34 patients needed High flow Oxygen or Invasive/Noninvasive Ventilation. Of these 34, 23 were IPD patients and 11 were OPD patients. In OPD patient group, 3 people needed pressure support ventilator and 7 needed high flow oxygen therapy (6-15litres/min) whereas in IPD study group 8 patients needed pressure support ventilator and 15 needed high flow oxygen therapy (650Litres/min). There was no significant change in length of stay of OPD and IPD patients. Because, IPD patient had more ICU stay due to more damaged lungs tissues, use of steroids to reduce the inflammation hence there was likely chances of developing more steroid induced 
myopathies, critical illness neuropathies and there was very gradual increase in physical exercises. Delay in pulmonary rehabilitation references due to hemodynamic instabilities or inconsistency in sessions due to complications like pneumothorax, pneumomediastinum. Hence, IPD group had more severe cases and complications during recovery time. Overall, all these factors have impacted the recovery in IPD group and statistically no significant difference was seen in both group's recovery time.

But it is observed that, IPD patients took lesser time to recover from Grade 1 to Grade $0.45 \%$ patients recovered in 1 week, $41 \%$ recovered in 2 weeks and $14 \%$ recovered in 3 weeks whereas, in OPD patients, only $5 \%$ recovered in 1 week, $11 \%$ in 2 weeks, $42 \%$ in 3 weeks, $25 \%$ in 4 weeks and $17 \%$ in 5 weeks. Hence, we can say when there is delayed pulmonary rehabilitation, the perceived exertion and dyspnoea takes more time to recover even if there is minimal or no need of oxygen supplementation.

Many studies have classified these patients as per their symptoms and radiological findings $15,19,24$ but we could not find any functional classification in these patients in recovery phase. This functional grading will be useful not only in COVID19 patients but also in other viral/ bacterial pneumonias, ARDS, secondary lung injuries due to systemic illness where we can expect functional impairments due to lung injury.

\section{Limitations of this study:}

We did not use any other functional scale or any quality-of-life scale for their assessment.

\section{Factors affected compliance in cardiopulmonary rehabilitation:-}

1. Patients having underlying comorbidities showed more disease related anxiety and fear during mobilization.

2. Post COVID complication like Pneumothorax, Pneumomediastinum.
3. Delayed reference for cardiopulmonary rehabilitation.

4. Previous physical activity level- Active ones were easy to rehabilitate than the sedentary.

5. Patients facing transport issues- Since they were dependent on family members physically and financially.

6. Telerehabilitation- Restricted use of resources.

7. Advanced age- Difficulty in gaining cooperation.

\section{CONCLUSION}

We can conclude that cardiopulmonary rehabilitation has a positive effect in recovery of COVID-19 patients. Also, the Post-Acute Lung Injury Functional Scale is an easy, effective, and appropriate tool for the functional assessment of COVID-19 patients as well as for patients who had undergone any kind of lung injury.

\section{ACKNOWLEDGEMENT}

We are thankful to our chest physicians Dr. Sunil Jaisingh and Dr. Ajay Godse and our physicians, Dr. Dhaval Dalal, Dr. Suraj P, Dr. Prashant patil, Dr. Gaurish Shetty, Dr. Meera R, Dr. Namrata Rao for referring patients. Also, Physicians of local hospitals Dr. Ajit Waingankar, Dr. Tanwar, Dr. Bhavin Shah who referred COVID-19 patients for cardiopulmonary rehabilitation in their recovery phase.

\section{Conflict of Interest: None}

\section{Source of Funding: None}

Ethical Approval: Approved

\section{REFERENCES}

1. Jiang F, Deng L, Zhang L, et al. Review of the Clinical Characteristics of Coronavirus Disease 2019 (COVID-19). J Gen Intern Med. 2020.

2. Huang C, Wang Y, Li X, et al. Clinical features of patients infected with 2019 
novel coronavirus in Wuhan, China. Lancet. 2020; 395:497-506.

3. Tian $\mathrm{S}, \mathrm{Hu} \mathrm{W}$, Niu L, et al. Pulmonary Pathology of Early-Phase 2019 Novel Coronavirus (COVID-19) Pneumonia in Two Patients with Lung Cancer. J Thorac Oncol. 2020; 15:700-704.

4. Xu Z, Shi L, Wang Y, et al. Pathological findings of COVID-19 associated with acute respiratory distress syndrome. Lancet Respir Med. 2020; 8:420-422.

5. Notice on Issuing Psychological Crisis Intervention Guidelines for the Novel Coronavirus Pneumonia Epidemic. The People's Republic of China: National Health Commission of the People's Republic of China, 2020 (Accessed January 26, 2020, at http://www.gov.cn/zhengce/zhengceku/20 20-01/27/content_5472433.html.)

6. Spruit MA, Singh SJ, Garvey C, et al. An official American Thoracic Society/European Respiratory Society statement: key concepts and advances in pulmonary rehabilitation. Am J Respir Crit Care Med. 2013;188: e13-64.

7. Charususin N, Gosselink R, Decramer M, et al. Randomised controlled trial of adjunctive inspiratory muscle training for patients with COPD. Thorax. 2018; 73:942-950.

8. Gordon CS, Waller JW, Cook RM, et al. Effect of Pulmonary Rehabilitation on Symptoms of Anxiety and Depression in COPD: A Systematic Review and MetaAnalysis. Chest. 2019; 156:80-91.

9. Calik-Kutukcu E, Arikan H, Saglam M, et al. Arm strength training improves activities of daily living and occupational performance in patients with COPD. Clin Respir J. 2017; 11:820-832.

10. Tarigan AP, Pandia P, Mutiara E, et al. Impact of Lower-Limb Endurance Training on Dyspnoea and Lung Functions in Patients with COPD. Open Access Maced J Med Sci. 2018; 6:23542358.

11. Hui DS, Joynt GM, Wong KT, et al. Impact of severe acute respiratory syndrome (SARS) on pulmonary function, functional capacity, and quality of life in a cohort of survivors. Thorax. 2005; 60:401-409.

12. Ng CK, Chan JW, Kwan TL, et al. Six month radiological and physiological outcomes in severe acute respiratory syndrome (SARS) survivors. Thorax. 2004; 59:889-891.

13. Lee $\mathrm{N}$, Hui $\mathrm{D}, \mathrm{Wu} \mathrm{A}$, et al. A major outbreak of severe acute respiratory syndrome in Hong Kong. N Engl J Med. 2003; 348:1986-1994.

14. Herridge MS, Cheung AM, Tansey CM, et al. One-year outcomes in survivors of the acute respiratory distress syndrome. $\mathrm{N}$ Engl J Med. 2003; 348:683-693.

15. Jiandani Mariya, COVID 19 and role of Physiotherapy. JAPI, april2021, volume 69; ISSN 0004-5772.

16. Joshi Mohan, Bharmal Ramesh, Sharma Alok, et al, principles of medical management of COVID-19- The MCGM experience, ISBN978-94-5457-294-7, 2021

17. Foye Patrick M, Bains. M, Lim S, Corticosteroid-Induced Myopathy. Drugs and Disease; Physical Medicine and Rehabilitation.

https://emedicine.medscape.com/article/31 3842-overview

18. Hodgson CL, Tipping CJ Physiotherapy management of intensive care unitacquired weakness. Journal of Physiotherapy, 2016, 63: 4-10

19. Qin L, Jing X, Qiu Z, Cao W, et al. Aging of immune system: immune signature from peripheral blood lymphocyte subsets in 1068 healthy adults. Aging (Albany NY). 2016 May; 8(5): 848-59.

20. Gulshan Sharma, James Goodwin, Effect of aging on respiratory system physiology and immunology, Clinical Interventions in Aging 2006:1(3) 253-260 () 2006

21. Suxin W, Qingjie Y, Shibing F, et al. Characteristics of lymphocyte subsets and cytokinesin peripheral blood of 123 hospitalized patients with 2019 novel coronavirus pneumonia (NCP). medRxiv. 2020. DOI: https://doi. org/10.1101/2020.02.10.20021832.

22. Mikuła T, Sapuła M, Jabłońska J, et al. Significance of heparin-binding protein and D-dimers in the early diagnosis of 
spontaneous bacterial peritonitis. Mediators Inflamm. 2018 Sep 30;2018: 1969108. doi: 10.1155/2018/1969108

23. Gris JC, Bouvier S, Cochery-Nouvellon E, et al. Fibrin- related markers in patients with septic shock: individual comparison of D-dimers and fibrin monomers impacts on prognosis. Thromb Haemost. 2011 Dec; 106(6): 1228-30.

24. Jiandani MP, Salagre SB, Kazi S, Iyer S, Patil P, Khot WY, Patil E, Sopariwala M. Preliminary Observations and Experiences of Physiotherapy Practice in Acute Care Setup of COVID 19: A Retrospective Observational Study. J Assoc Physicians India. 2020 Oct;68(10):18-24. PMID: 32978920 .

How to cite this article: Gosavi N, Karande B, Godse A et.al. Assessment of post COVID-19 rehabilitation programme using post-acute lung injury functional scale. Int $J$ Health Sci Res. 2021; 11(10): 311-321. DOI: https://doi. org/10.52403/ijhsr.20211041 